\title{
Fluorescence lifetime imaging by using time-gated data acquisition
}

\author{
Vadim Y. Soloviev, ${ }^{1, \star}$ Khadija B. Tahir, ${ }^{2}$ James McGinty, ${ }^{2}$ Dan S. Elson, ${ }^{2}$ Mark A. A. Neil, ${ }^{2}$ \\ Paul M. W. French, ${ }^{2}$ and Simon R. Arridge ${ }^{1}$ \\ ${ }^{1}$ Department of Computer Science, University College London, Gower Street, London WC1E 6BT, UK \\ ${ }^{2}$ Imperial College Photonics, Blackett Laboratory, Prince Consort Road, London SW7 2BW, UK \\ ${ }^{\star}$ Corresponding author: v.soloviev@cs.ucl.ac.uk \\ Received 14 February 2007; revised 4 July 2007; accepted 23 July 2007; \\ posted 30 July 2007 (Doc. ID 80108); published 10 October 2007
}

\begin{abstract}
The use of the time gating technique for lifetime reconstruction in the Fourier domain is a novel technique. Time gating provides sufficient data points in the time domain for reliable application of the Fourier transform, which is essential for the time deconvolution of the system of the integral equations employed in the reconstruction. The Fourier domain telegraph equation is employed to model the light transport, which allows a sufficiently broad interval of frequencies to be covered. Reconstructed images contain enough information needed for recovering the lifetime distribution in a sample for any given frequency within the megahertz-gigahertz band. The use of this technique is essential for recovering time-dependent information in fluorescence imaging. This technique was applied in reconstruction of the lifetime distribution of four tubes filled with Rhodamine 6G embedded inside a highly scattering slab. Relatively accurate fluorescence lifetime reconstruction demonstrates the effectiveness and the potential of the proposed technique. (C) 2007 Optical Society of America

OCIS codes: $290.0290,290.7050,170.0170,170.3010$.
\end{abstract}

\section{Introduction}

Lifetime imaging has many applications in biology and medicine, for example, the lifetime of luminophor in tissue can provide functional information such as $\mathrm{pH}$, enzyme, and oxygenation [1-3]. This paper explores the potential of 3D fluorescence lifetime tomography utilizing wide-field time-gated image data. Fluorescence lifetime imaging and tomography in scattering media has previously been studied in the time domain [4-6] but most of the reported work has employed frequency domain lifetime imaging [7-12]. If the amplitude of the excitation light is sinusoidally modulated, a phase shift between the amplitude of excitation light and the amplitude of re-emitted fluorescent light provides additional information, which allows reconstruction of lifetimes. Because typical fluorescence lifetimes are short, the phase shift between the excitation and fluorescent light is very

0003-6935/07/307384-08\$15.00/0

(C) 2007 Optical Society of America small for modulation frequencies below several megahertz. Therefore, lifetime reconstruction requires using higher frequencies. However, frequencies of amplitude modulation of up to $1 \mathrm{GHz}$ and higher are not technically achievable yet as well as not practical due to exponential damping of the ac component with the frequency. That is to say, the light transport at frequencies of the amplitude modulation in the gigahertz band and higher behaves almost identically to the steady state case.

Time-domain data acquisition is an alternative to frequency domain in fluorescence lifetime imaging. A fluorophor is excited from the boundary by an ultrashort pulse of light, and the fluorescent intensity is recorded as a function of time on the surface of a scattering volume. Experimentally it is achievable by using the time gating technique $[13,14]$ when the light is collected within a very short exposure time. Measured signals are then Fourier transformed to give complex functions, which form a data set for reconstruction. The very large interval of frequencies makes it possible to pick any frequency for recon- 
struction. In addition, the experimental noise in the time domain is mapped into higher frequencies, which results in much higher signal-to-noise ratio for frequencies below a few gigahertz. In this study we apply this approach for experimental lifetime image reconstruction. It should be mentioned that similar approaches were suggested earlier but without experimental validations $[15,16]$.

In this paper the telegraph equation (TE) is employed to model the light transport in turbid media in the Fourier domain. The TE assumes finite propagation speed and contains the well-known diffusion approximation as a limiting case. The TE is more suitable for such type of techniques due to its better accuracy for higher frequencies than the diffusion approximation [17,18].

In terms of data acquisition, our approach employs noncontact imaging, which may provide higher accuracy than earlier tomographic schemes that use a number of discrete light guides. For a noncontact imaging, a CCD camera is placed at some distance from the scattering volume. Each pixel of the CCD camera absorbs photons escaping from the imaging surface. We note that, although noncontact imaging provides a large number of measurements acquired for every position of an excitation source (the typical number of pixels is $\sim 10^{5}$ ), each measurement data set must be mapped onto the surface of a scattering object prior to image reconstruction by taking into account the optical system and geometry $[19,20]$.

This paper presents reconstruction of the quantum yield and the lifetime distribution from the experimental data set by use of the time gating technique for what we believe to be the first time. The time gating technique provides enough data points in the time domain needed for the application of the Fourier transform. The use of the Fourier transform is essential for the time deconvolution of the system of the integral equations employed for the lifetime reconstruction. The Fourier transform is more efficient and suitable for the time gating technique than the Laplace transform suggested by Gao et al. [16]. Reconstructed complex valued functions depending on the quantum yield and the lifetime contain all the information needed for recovering the lifetime distribution in a sample for any given frequency within the megahertz-gigahertz frequency band.

The paper is organized as follows. To make the paper self-contained we briefly outline the basic theory of the light transport in highly scattering media and the associated inverse problem in the next section with an emphasis on experimentally observable quantities. A photon as a quantum object is not observable unless it is absorbed. Quantities used as the dataset for the reconstruction are derived from the absorbed energy of light by the CCD camera. A phantom and an experimental implementation of the time gating technique is described in Section 3. Section 4 presents reconstruction results, discussions, and conclusions.

\section{Methodology}

\section{A. Experimentally Observable Quantities}

A radiation field in space is described by the distribution of the intensity of radiation with respect to frequency, to space, and to the direction of the radiant energy transfer. If we consider radiation as a collection of particles (photons), then the field can be described by a photon distribution function $f$, which is, to a large extent, analogous to any other particle distribution function. Let $f(\nu, \mathbf{r}, \mathbf{s}, t) \mathrm{d} \nu \mathrm{d}^{3} \mathbf{r d} \mathbf{s}$ be the number of photons in the frequency interval $(v, v+\mathrm{d} v)$, contained at the time $t$ in the volume element $\mathrm{d}^{3} \mathbf{r}$ about the point $\mathbf{r}$, and having a direction of motion within an element of solid angle ds $=$ $\sin \theta \operatorname{d} \varphi d \theta$ about a unit vector $\mathbf{s}=(\sin \theta \cos \varphi$, $\sin \theta \sin \varphi, \cos \theta)^{T}$, where $\theta$ is the polar angle and $\varphi$ is azimuthal angle. Each photon possesses an energy $h \nu$ and moves with the speed $c$, where $h$ is the Planck constant. Hence the quantity

$$
I_{\nu}(\mathbf{r}, \mathbf{s}, t) \mathrm{d} \nu \mathrm{d} \mathbf{s}=h \nu c f(\nu, \mathbf{r}, \mathbf{s}, t) \mathrm{d} \nu \mathrm{d} \mathbf{s}
$$

represents a radiant energy in the spectral interval $\mathrm{d} \nu$, passing per unit time through a unit area, with directions of energy propagation contained within the element of solid angle ds, about the vector $\mathbf{s}$. The area is allocated at the point $\mathbf{r}$ and oriented perpendicular to $\mathbf{s}$. The quantity $I_{v}$ is called the spectral radiation intensity. The radiation field is essentially fully defined by specifying either the function $I_{v}$ or $f$. The radiant energy of frequency $v$ included in a unit spectral interval of frequency and contained in a unit volume at the point $\mathbf{r}$ at the time $t$ is termed the spectral radiant energy density (the energy density for short) and is given by

$$
U_{v}(\mathbf{r}, t)=h v \int_{(4 \pi)} f \mathrm{~d} \mathbf{s}=\frac{1}{c} \int_{(4 \pi)} I_{v} \mathrm{~d} \mathbf{s} .
$$

The number of photons in the frequency interval $\mathrm{d} v$ exiting the unit area on the surface of a scattering volume per unit time within an element of solid angle ds, i.e., the particle flux $\left[\mathrm{mm}^{-2} \mathrm{~s}^{-1}\right]$, is equal to $c(\mathbf{n} \cdot \mathbf{s}) f \mathrm{~d} \mathbf{s}$, where $\mathbf{n}$ is the outward normal. Therefore, the number of photons $\mathrm{d} N$ escaping the scattering volume through the area $d S$ within the time interval $\mathrm{d} t$ and within an element of solid angle $\mathrm{d} \mathbf{s}$ about $\mathbf{s}$ is given by $\mathrm{d} N=f \mathrm{~d}^{3} \mathbf{r} \mathrm{d} \mathbf{s}$, where $\mathrm{d}^{3} \mathbf{r}=(\mathbf{n} \cdot \mathbf{s}) c \mathrm{~d} t \mathrm{~d} S$. Next, let us assume that photons moving from the surface of the scattering volume within $\mathrm{d}^{3} \mathbf{r}$ are absorbed by an area $d S_{p}$ of the distant absorbing surface such as a CCD camera. Considering the reverse process we find the number of photons absorbed by the area $\mathrm{d} S_{p}$ within $\mathrm{d} t$ and within $\mathrm{d} \mathbf{s}_{p}=-\mathrm{d} \mathbf{s}$ about the unit vector $\mathbf{s}_{p}=-\mathbf{s}$ in the form

$$
\mathrm{d} N_{p}=f \mathrm{~d}^{3} \mathbf{r}_{p} \mathrm{~d} \mathbf{s}_{p}, \quad \mathrm{~d}^{3} \mathbf{r}_{p}=\left(\mathbf{n}_{p} \cdot \mathbf{s}_{p}\right) c \mathrm{~d} t \mathrm{~d} S_{p},
$$


where $\mathbf{n}_{p}$ is outward normal to the absorbing surface and $\mathbf{n}_{p} \cdot \mathbf{s}_{p} \geq 0$.

The total number of photons absorbed by $\mathrm{d} S_{p}$ within the time interval $\mathrm{d} t$ is obtained by summing all photons arriving at a pixel of the CCD camera at $\mathbf{r}_{p}$ from the visible part of the emitting surface within the solid angle $\Omega_{p}$ [19]:

$N_{p}(t)=c \mathrm{~d} t \mathrm{~d} S_{p} \int w\left(\mathbf{s}_{p}, \mathbf{s}_{0}\right) f\left(\mathbf{r}, \mathbf{s}_{p}, t\right)\left(\mathbf{n}_{p} \cdot \mathbf{s}_{p}\right) \mathrm{d} \mathbf{s}_{p}$,

where the weighting function $w\left(\mathbf{s}_{p}, \mathbf{s}_{0}\right)$ describes the optical system. An optical system consisting of a lens placed at sufficient distance from the emitting surface can be approximated by a pinhole. A pinhole assumption results in a sharply peaked weighting function $w\left(\mathbf{s}_{p}, \mathbf{s}_{0}\right)$ about a direction $\mathbf{s}_{0}$ along the line connecting each pixel of the CCD camera to the emitting surface through a pinhole. Therefore, one can bring out all slowly varying functions from the integral in Eq. (4) at $\mathbf{s}_{0}$. Considering the direct relationship between the sample emission and the detector surfaces (which is parallel in our case), one can assume that $w\left(\mathbf{s}_{p}, \mathbf{s}_{0}\right)$ depends only on $\cos \vartheta_{0}=\mathbf{s}_{p} \cdot \mathbf{s}_{0}$ and, consequently, setting $w=\delta\left(\cos \vartheta-\cos \vartheta_{0}\right)$ obtain

$$
N_{p}(t) \simeq 2 \pi c \mathrm{~d} t \mathrm{~d} S_{p} f\left(\mathbf{r}, \mathbf{s}_{0}, t\right)\left(\mathbf{n}_{p} \cdot \mathbf{s}_{0}\right) .
$$

Because not all absorbed photons are counted, it is useful to introduce a camera's gating function $g_{v}(t)$ in the frequency interval $(v, v+\mathrm{d} v)$. The camera's gating function describes the camera's response to the $\delta$-function input and has a dimension of inverse time. Thus the number of counted photons, denoted by $N_{p}{ }^{\prime}$, is given by the time convolution of the number of absorbed photons, $N_{p}(t)$, with the gating functions $g_{v}(t)$. An assumption that $g_{v}(t)$ has finite support about $t$ allows the integration to be extended over the entire time domain:

$$
N_{p}^{\prime}(t)=\int^{\infty} g_{v}\left(t-t^{\prime}\right) N_{p}\left(t^{\prime}\right) \mathrm{d} t^{\prime} .
$$

By means of Eq. (1) this number is converted to the energy $E_{p}{ }^{\prime}(t)=h \nu N_{p}{ }^{\prime}(t)$ recorded by a pixel of the CCD camera of area $\Delta S_{p}$ within the finite but small exposure time $\Delta t$ and can be regarded as the observable quantity in the time domain,

$$
E_{p}{ }^{\prime}(t) \simeq 2 \pi \Delta t \Delta S_{p}\left(\mathbf{n}_{p} \cdot \mathbf{s}_{0}\right) \int_{-\infty}^{\infty} g_{v}\left(t-t^{\prime}\right) I_{v}\left(\mathbf{r}, \mathbf{s}_{0}, t^{\prime}\right) \mathrm{d} t^{\prime},
$$

as well as in the Fourier domain ( $\omega)$,

$$
E_{p}{ }^{\prime}(\omega) \simeq 2 \pi \Delta t \Delta S_{p}\left(\mathbf{n}_{p} \cdot \mathbf{s}_{0}\right) g_{v}(\omega) I_{v}\left(\mathbf{r}, \mathbf{s}_{0}, \omega\right) .
$$

Technically, each pixel of the CCD camera records a signal proportional to $E_{p}{ }^{\prime}(t)$ at the finite number of points $t=t_{n}$. Recorded signals are then Fourier transformed to give complex functions $E_{p}{ }^{\prime}\left(\omega_{n}\right)$, which form the data set for the lifetime image reconstruction.

When the exposure time is very small the gating function $g_{v}$ can be quite accurately approximated by the step function defined as: $g_{v}(t)=g_{0} / \Delta t$ when $t_{d}$ $<t<t_{d}+\Delta t$ and zero otherwise, where a dimensionless constant $g_{0}$ describes how the absorbed energy is transformed to a recorded signal (assuming linear dependence), and $t_{d}$ is the time delay with respect to excitation time. The Fourier transform of the step function is expressed in terms of a sinc function:

$$
g_{v}(\omega)=g_{0} \exp \left[-i \omega\left(t_{d}+\Delta t / 2\right)\right] \operatorname{sinc}(\omega \Delta t / 2) .
$$

The exposure time in our experiments was set to $\Delta t$ $\simeq 5 \times 10^{-10} \mathrm{~s}$. For the diffusion regime $\omega \ll 10^{8} \mathrm{~Hz}$ and the delay time $t_{d} \sim \Delta t$ the following holds: $\omega \Delta t \ll 1$ and $\omega t_{d} \ll 1$, and, therefore, $g_{v}(\omega) \simeq g_{0}$. For the frequencies $\omega \sim 10^{8} \mathrm{~Hz}$ and higher the whole expression Eq. (9) must be used.

\section{B. Radiative Transfer Equation and Its Approximations}

We employ the TE approximation to the radiative transfer equation for modeling the light transport in the turbid medium. The TE is the result of consequent simplifications made to the radiative transfer equation according to the conditions assumed to be valid for the studied physical system $[21,22]$. In the Fourier domain the radiative transfer equation describing the evolution of the intensity $I_{v}$ due to an ultrashort pulse of light at $t_{0}$ reads as

$$
\begin{aligned}
\left(\mathbf{s}, \nabla I_{v}\right)= & -\gamma_{0} I_{v}+\frac{\mu_{s}}{4 \pi} \int p\left(\mathbf{s}^{\prime} \cdot \mathbf{s}\right) I_{v}\left(\mathbf{s}^{\prime}\right) \mathrm{d} \mathbf{s}^{\prime} \\
& +p\left(\mathbf{s}_{s} \cdot \mathbf{s}\right) S_{0}^{(4 \pi)} \\
S_{0}= & \varpi_{0} N_{0} h v \Lambda\left(\mathbf{r}-\mathbf{r}_{0}\right) \exp \left[-i \omega\left(t_{0}+\left|\mathbf{r}-\mathbf{r}_{0}\right| / c\right)\right],
\end{aligned}
$$

$$
\Lambda\left(\mathbf{r}-\mathbf{r}_{0}\right)=\frac{\mu_{t r}(\mathbf{r})}{4 \pi\left|\mathbf{r}-\mathbf{r}_{0}\right|^{2}} \exp \left(-\int_{0}^{\left|\mathbf{r}-\mathbf{r}_{0}\right|} \mu_{t r}\left(\mathbf{r}^{\prime}\right) \mathrm{d} l^{\prime}\right),
$$

where $N_{0}$ is the number of injected photons. The scattering coefficient is denoted by $\mu_{s}$ and the absorption by $\mu_{a} ; \mu_{t r}=\mu_{s}+\mu_{a}$ is the transport coefficient $\left(1 / \mu_{t r}=l\right.$, where $l$ is the free path length); $\varpi_{0}$ $=\mu_{s} / \mu_{t r}$ is the albedo of a single scattering event; $\gamma_{0}$ is given by

$$
\gamma_{0}=\mu_{t r}\left(1+i \omega / c \mu_{t r}\right),
$$

and $p\left(\mathbf{s}^{\prime} \cdot \mathbf{s}\right)$ is the phase function. The unit vector $\mathbf{s}_{s}$ originates at the point source and directs toward the observation point $\mathbf{r}=(x, y, z)^{T}$, while the coordinates of a point source are denoted by $\mathbf{r}_{0}=\left(x_{0}, y_{0}, z_{0}\right)^{T}$. The 
integration in Eq. (12) is performed along the straight line connecting points $\mathbf{r}_{0}$ and $\mathbf{r}$.

The source term in Eq. (11) describes only the scattered (diffusive) photons incoming from the point-like source, while ballistic photons are excluded [23]. Thus, for the perfectly absorbing medium $\varpi_{0}=0$ as well as for the nonscattering medium $l=\infty$, the source term $S_{0}$ disappears. The amplitude modulated light transport model is obtained by convolving the source term, Eq. (11), with a function governing the amplitude modulation, say $\psi\left(t_{0}\right)$, with respect to $t_{0}$ [i.e., $\left.\exp \left(-i \omega t_{0}\right) \Rightarrow \psi(\omega)\right]$. The physical meaning of the source term in Eq. (11) becomes clear in the time domain:

$$
\begin{gathered}
S_{0}(t)=\varpi_{0} N_{0} h v \Lambda_{0}\left(\mathbf{r}-\mathbf{r}_{0}\right) \delta\left(t-t_{0}-\left|\mathbf{r}-\mathbf{r}_{0}\right| / c\right), \\
\Lambda_{0}\left(\mathbf{r}-\mathbf{r}_{0}\right) \simeq \mu_{t r} \exp \left(-\mu_{t r}\left|\mathbf{r}-\mathbf{r}_{0}\right|\right) / 4 \pi\left|\mathbf{r}-\mathbf{r}_{0}\right|^{2},
\end{gathered}
$$

where a constant $\mu_{t r}$ is assumed in the neighborhood of $\mathbf{r}_{0}$. The function $\Lambda_{0}\left(\mathbf{r}-\mathbf{r}_{0}\right)$ in Eq. (14) can be regarded as a distribution function of ballistic photons scattered for the first time. Its first moment in infinite space gives unity. The second moment gives $1 / \mu_{t r}$, i.e., the mean free path $l$. The $\delta$ function in Eq. (14) describes the time delay of ballistic photons arriving at $\mathbf{r}$. In a highly scattering medium with low absorption the source term $S_{0}$ can be appreciably simplified. First, at the distances where $\mu_{t r}\left|\mathbf{r}-\mathbf{r}_{0}\right| \gg 1$ the distribution function $\Lambda_{0}\left(\mathbf{r}-\mathbf{r}_{0}\right)$ is replaced with $\delta(\mathbf{r}$ $-\mathbf{r}_{0}$ ). Then, assuming that all injected photons are scattered immediately at $t_{0}$ in the neighborhood of $\mathbf{r}_{0}$ the time delayed $\delta$ function is replaced by $\delta\left(t-t_{0}\right)$. Therefore, the source function in the Fourier domain describing the pulse of light of duration $0<t_{0}<\delta t$ can be approximated by

$$
S_{0} \simeq \varpi_{0} N_{0} h \nu \delta\left(\mathbf{r}-\mathbf{r}_{0}\right) \exp (-i \omega \delta t / 2) \operatorname{sinc}(\omega \delta t / 2) .
$$

Taking $\delta t \sim 10 \mathrm{ps}$ and considering frequencies below several gigahertz we can simplify the source function even further by replacing $\exp (-i \omega \delta t / 2) \operatorname{sinc}(\omega \delta t / 2)$ by 1 .

The telegraph equation approximation to the radiative transfer results by retaining the first two terms in the Fourier power series of the phase function and is obtained by taking the first two moments of the radiative transfer equation. If the energy flux $\mathbf{H}$ is defined as

$$
\mathbf{H}_{v}(\mathbf{r}, \omega)=\int_{(4 \pi)} I_{v}(\mathbf{r}, \mathbf{s}, \omega) \mathbf{s} \mathrm{d} \mathbf{s},
$$

then the system of two coupled differential equations in the Fourier domain $(\omega)$ reads as

$$
\begin{array}{ll}
\nabla \cdot \mathbf{H}_{v}+c q U_{v}=S_{0}, & \mathbf{H}_{v} \simeq-\left(c / 3 \gamma_{1}\right) \nabla U_{v}, \\
q=\mu_{a}\left(1+i \omega / c \mu_{a}\right), & \gamma_{1}=\gamma_{0}-\mu_{s} g
\end{array}
$$

and $g$ is the average cosine. By eliminating the energy flux from Eq. (17), the Helmholtz equation, the Fourier image of the telegraph equation, is obtained. The diffusion approximation (DA) results by neglecting $i \omega / c \mu_{t r}$ in $\gamma_{1}$.

Robin type boundary conditions for the TE or DA follow from the approximate expression of the intensity,

$$
I_{v} \simeq \frac{c}{4 \pi} U_{v}+\frac{3}{4 \pi} \mathbf{s} \cdot \mathbf{H}_{v},
$$

by taking into account Eq. (17) together with the conditions $\left.I(\mathbf{n} \cdot \mathbf{s}<0)\right|_{\partial V}=0$ on the surface of the scattering volume, where $\mathbf{n}$ is the surface's outward normal. Making use of Eq. (19) at the boundary of the scattering volume $\partial V$ one can rewrite Eq. (8) in the form:

$$
E_{p}{ }^{\prime}(\omega) \simeq c \Delta t \Delta S_{p}\left(\mathbf{n}_{p} \cdot \mathbf{s}_{0}\right) g_{v}(\omega) U_{v}(\mathbf{r}, \omega) .
$$

\section{Inverse Problem: the Time Domain Versus the Fourier} Domain

An assumption that all nonlinear effects in the emission light transport are negligible makes the mathematical description of the emission photon transport analogous to the excitation one except for different values of optical parameters and a source function. The number of created fluorescent photons $N_{v^{*}}$ [instead of $N_{0}$ in Eq. (11)] is proportional to the time convolution of the absorbed excitation energy density $\left(\mu_{a} / \mu_{s}\right) U_{v}$ and the $\delta$ response of the fluorophor:

$$
q_{v^{*}}\left(\mathbf{r}^{\prime}, t-t^{\prime}\right)=q_{0} H\left(t-t^{\prime}\right) \int_{0}^{\infty} F\left(\mathbf{r}^{\prime}, \tau^{\prime}\right) \exp \left(-\frac{t-t^{\prime}}{\tau^{\prime}}\right) \frac{\mathrm{d} \tau^{\prime}}{\tau^{\prime}},
$$

where $H\left(t-t^{\prime}\right)$ is the Heaviside step function, $q_{0}$ is the quantum yield, $\tau$ is the lifetime, and $F\left(\mathbf{r}^{\prime}, \tau\right)$ is the lifetime distribution function. Thus, the number of fluorescence photons created by a volume $\mathrm{d}^{3} \mathbf{r}^{\prime}$, each possessing an energy $h v^{*}$, is given in the form of a convolution:

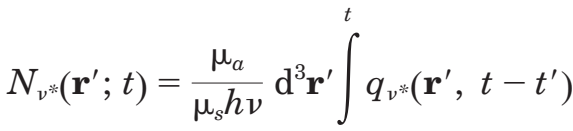

$$
\begin{aligned}
& \times U_{v}\left(\mathbf{r}^{\prime}, \stackrel{t_{0}}{\mathbf{r}_{0}} ; t^{\prime}-t_{0}\right) \mathrm{d} t^{\prime} .
\end{aligned}
$$

The lower limit of the integral in Eq. (22) can be extended over the entire history of the system, i.e., to $-\infty$, because $U_{v}\left(t^{\prime}-t_{0}\right)=0$ when $t^{\prime}<t_{0}$, and the upper limit can be extended to $\infty$ due to properties of the Heaviside function.

For the further analysis it is convenient to introduce the Green function. The fluorescent Green function in the time domain $G_{p^{*}}\left(\mathbf{r}, \mathbf{r}^{\prime} ; t-t^{\prime}\right)$ is found by applying the inverse Fourier transform to the solu- 
tion of the boundary value problem,

$$
\nabla\left(1 / 3 \gamma_{1}^{*}\right) \nabla G_{v^{*}}-q^{*} G_{v^{*}}=-\mu_{s}^{*} \delta\left(\mathbf{r}-\mathbf{r}^{\prime}\right) \exp \left(-i \omega t^{\prime}\right),
$$

subject to Robin boundary conditions. The parameters $\gamma_{1}{ }^{*}, \mu_{s}{ }^{*}$, and $q^{*}$ are the fluorescent analogs of $\gamma_{1}$, $\mu_{s}$, and $q$. The scattering coefficient $\mu_{s}^{*}$ appearing in the source function expresses the fact that only scattered photons are considered. Note that the Green function defined by Eq. (23) has a dimension $\mathrm{mm}^{-3}$ $\mathrm{s}^{-1}$ in the time domain and $\mathrm{mm}^{-3}$ in the Fourier domain. The number of scattered fluorescence photons $N_{v^{*}}(\mathbf{r}, t)$ is given by the time convolution of the Green function with the number of created fluorescent photons:

$N_{v^{*}}(\mathbf{r} ; t)=\mathrm{d}^{3} \mathbf{r} \int^{t} N_{\nu^{*}}\left(\mathbf{r}^{\prime}, t^{\prime}\right) G_{\nu^{*}}\left(\mathbf{r}, \mathbf{r}^{\prime} ; t-t^{\prime}\right) \mathrm{d} t^{\prime}$,

where the integration can be extended over the entire time domain. Insertion of Eq. (22) into Eq. (24) together with Eqs. (5)-(7) results in a system of Fredholm integral equations of the first kind:

$$
\begin{aligned}
E_{p}{ }^{\prime}(\mathbf{r} ; t)= & c \Delta t \Delta S_{p}\left(\mathbf{n}_{p} \cdot \mathbf{s}_{0}\right)\left(v^{*} / v\right) \int_{V} \mathrm{~d}^{3} \mathbf{r}^{\prime}\left(\mu_{a} / \mu_{s}\right) \\
& \times \iiint_{-\infty}^{\infty} \mathrm{d} t^{\prime} \mathrm{d} t^{\prime \prime} \mathrm{d} t^{\prime \prime \prime} g_{v^{*}}\left(t-t^{\prime}\right) q_{v^{*}}\left(\mathbf{r}^{\prime} ; t^{\prime \prime}-t^{\prime \prime \prime}\right) \\
& \times G_{v^{*}}\left(\mathbf{r}, \mathbf{r}^{\prime} ; t^{\prime}-t^{\prime \prime}\right) U_{v}\left(\mathbf{r}^{\prime}, \mathbf{r}_{0} ; t^{\prime \prime \prime}-t_{0}\right),
\end{aligned}
$$

where $p$ runs through all pixels of images taken by CCD camera; $\mathbf{r}_{0}$ runs through all source positions; and $g_{v^{*}}(t)$ is the gating function in the frequency interval $\left(v^{*}, v^{*}+\mathrm{d} v^{*}\right)$. An additional time convolution will be present in Eq. (25) in the case of a sufficiently long excitation pulse. The inverse problem formulated as the reconstruction of the quantum yield and the lifetime in the time domain on the basis of a system of equations of Eq. (25) type is almost intractable due to the triple time convolution. That is to say, the inverse problem is three-dimensional in time. However, the system becomes appreciably simpler in the Fourier domain where it reads as

$$
\begin{aligned}
& E_{p}{ }^{\prime}(\mathbf{r} ; \omega) \simeq c \Delta t \Delta S_{p}\left(\mathbf{n}_{p} \cdot \mathbf{s}_{0}\right)\left(\nu^{*} / v\right) \int_{V} \mathrm{~d}^{3} \mathbf{r}^{\prime}\left(\mu_{a} / \mu_{s}\right) \\
& \times G_{v^{*}}\left(\mathbf{r}, \mathbf{r}^{\prime} ; \omega\right) U_{v}\left(\mathbf{r}^{\prime}, \mathbf{r}_{0} ; \omega\right) Q_{0}\left(\mathbf{r}^{\prime} ; \omega\right), \\
& Q_{0}(\mathbf{r} ; \omega)=g_{v^{*}}(\omega) q_{0}(\mathbf{r}) \int_{0}^{\infty} \frac{F\left(\mathbf{r}, \tau^{\prime}\right) \mathrm{d} \tau^{\prime}}{1+i \omega \tau^{\prime}} .
\end{aligned}
$$

An assumption that the lifetime distribution function is sharply peaked at $\tau(\mathbf{r})$ results in

$$
Q_{0}(\mathbf{r} ; \omega) \simeq \frac{g_{v^{*}}(\omega) q_{0}(\mathbf{r})}{1+i \omega \tau(\mathbf{r})}
$$

where $\int F\left(\tau^{\prime}\right) \mathrm{d} \tau^{\prime}=1$ has been used.

The system of Eq. (26) is further discretized and solved numerically in the Fourier domain on the adaptive Cartesian grid [15,24] by use of the conjugate gradient method with early termination of the iterative process, which has a regularization effect. The number of iterations is found empirically from the discrepancy principle [25].

\section{Materials and Experimental Methods}

\section{A. Phantom}

For this experiment a solid phantom of slab geometry $120 \times 75 \times 40 \mathrm{~mm}$ shown in Fig. 1 was built using a solution of epoxy (MY753) and hardener (XD716) from Robnor Resins Ltd. mixed with the scattering particles $\left(\mathrm{TiO}_{2}\right)[26,27]$. The resin has a refractive index of 1.547 , the $\mathrm{TiO}_{2}$ particles (Tiranti Ltd., London), has an average diameter of $0.25 \mu \mathrm{m}$ and its refractive index is 2.58 . The following values of optical parameters were measured [28]: $\mu_{s}=$ $2.0016 \mathrm{~mm}^{-1} ; g=0.5580 ; \mu_{a}=0.0008 \mathrm{~mm}^{-1}$. We assume that the optical parameters of the medium
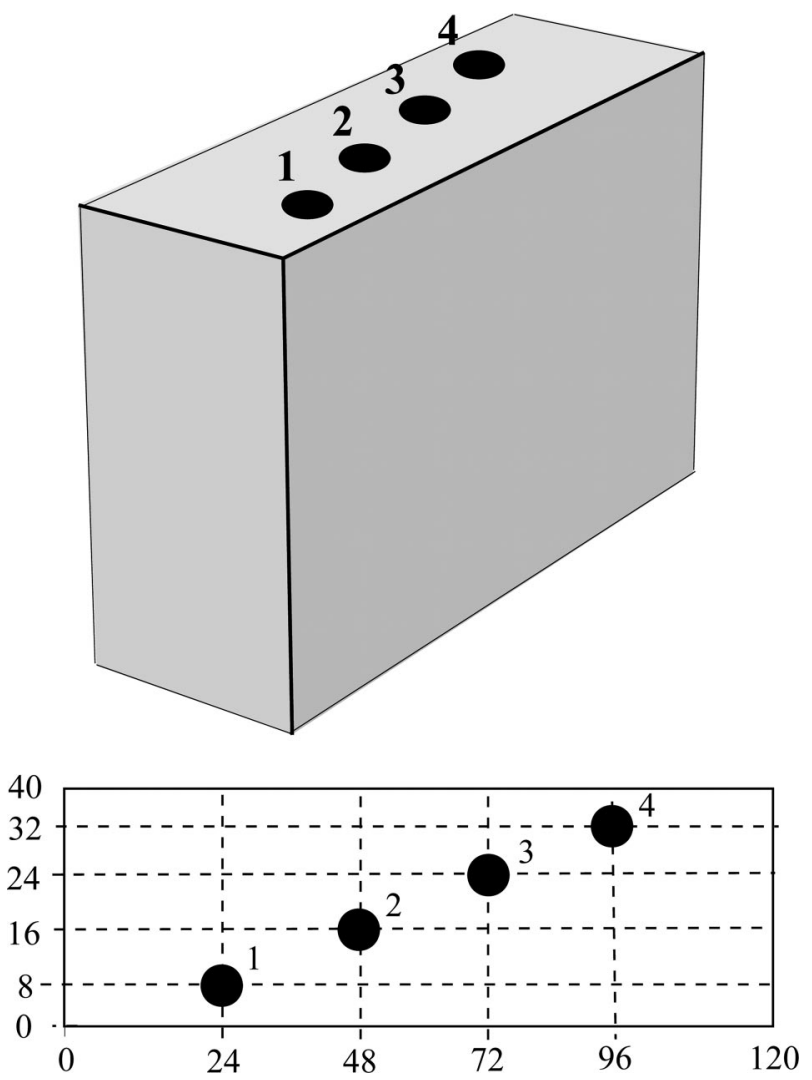

Fig. 1. Phantom and the positions of tubes filled with Rhodamine 6G. 
with respect to excitation and fluorescence are the same. This assumption is reasonable considering the corresponding wavelength, which results in the difference between the corresponding optical parameters being indistinguishable from the experimental error. The slab was drilled with four wells each of $8 \mathrm{~mm}$ diameter. These wells had the same optical properties but mixed with Rhodamine 6G dye as the fluorophor.

\section{B. Experimental Setup and Method}

The illumination was provided by a commercial supercontinuum source (SC450-2, Fianium Ltd.), producing a continuum from 450 to $2000 \mathrm{~nm}$ with a spectral density up to $5 \mathrm{~mW} / \mathrm{nm}$ at a repetition rate of $50 \mathrm{MHz}$. An electronically tunable spectral selection arm [29] was incorporated in the setup to select an appropriate excitation wavelength. The diagram of the experimental setup is shown in Fig. 2. Briefly, following angular dispersion at the prism, $\mathrm{P}$, the continuum was collimated by an achromatic lens ( $f=180 \mathrm{~mm}, 01 \mathrm{LAO} 139$, Melles Griot) positioned one focal length from the prism. A motorized triangularshaped aperture located directly in front of a mirror, M3, provided spectral selection and bandwidth control (horizontal and vertical adjustment, respectively). After reflection back through the lens-prism arrangement (removing the angular dispersion), the selected spectral range was picked off by a knife-edge mirror M4. To efficiently excite the fluorescent cylinders, $25 \mathrm{~mW}$ at a wavelength of $525 \pm 15 \mathrm{~nm}$ was direct toward the sample using M5, resulting in a "source" diameter of $\sim 5 \mathrm{~mm}$ with a pulse duration of $\sim 10$ ps.

Time-gated diffuse transmitted fluorescence images were acquired using a gated optical intensifier

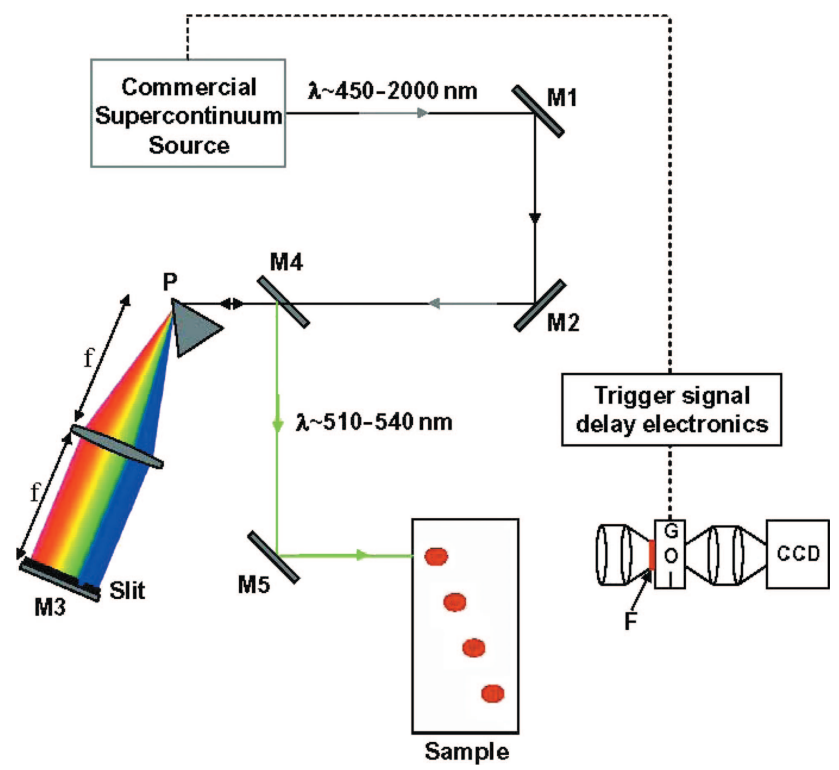

Fig. 2. (Color online) Experimental setup. M1-M5, mirrors; P, prism; F, long pass filter. Mirror M3 has a triangular-shaped slit positioned directly in front of it. Sample is mounted on a motorized translation stage to produce different source positions.

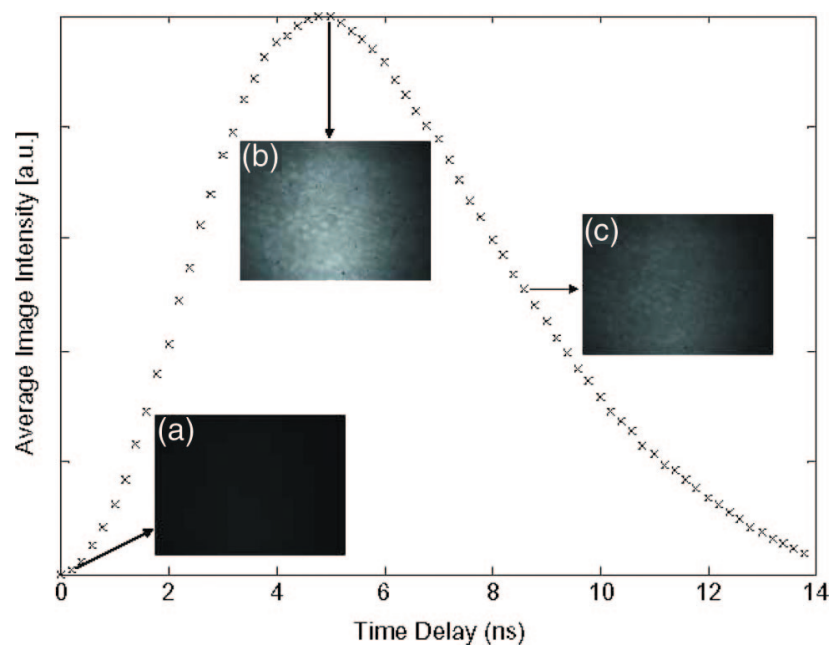

Fig. 3. (Color online) Graph showing the average image intensity as a function of time-gated image delay, including gated images at delays of (a) 0 , (b) 5 , and (c) 8.6 ns.

(GOI, Kentech Instruments Ltd.) optically coupled to a CCD (OrcaER, Hamamatsu Photonics). The GOI can produce time gates ranging in duration from $<100 \mathrm{ps}$ up to $1 \mathrm{~ns}$ at repetition rates kilohertz to $1 \mathrm{GHz}$. For the acquisitions described here the gate width was set to $600 \mathrm{ps}$ with the GOI triggered at the repetition rate of the laser. The transmitted fluorescence signal was imaged onto the photocathode of the GOI through a $550 \mathrm{~nm}$ long pass filter (550GY25, Comar) using a camera lens (CC21211, Cosmicar/ Pentax), imaging $3.0 \times 2.3 \mathrm{~cm}$ of the back surface of the sample.

During an acquisition, 70 time-gated images at 200 ps intervals, controlled by delay electronics (Trigger Delay Unit, Kentech Instruments Ltd.), were recorded, covering a sampling extent of $13.8 \mathrm{~ns}$ as indicated in Fig. 3. The gain voltage and integration time were fixed at $950 \mathrm{v}$ and $0.1 \mathrm{~s}$ on the GOI and CCD, respectively. The sample was then translated in $4 \mathrm{~mm}$ intervals to generate data sets for a number of source positions, ranging from $8 \mathrm{~mm}$ either side of the first and last fluorescent cylinders.

\section{Results and Discussions}

Quantum yield was reconstructed from the steady state case $\omega=0$. Slice at $y=25 \mathrm{~mm}$ is shown in Fig. 4. The overall spatial accuracy of the reconstruction is $\sim 2-3 \mathrm{~mm}$. The spatial resolution of the tube closest to the camera is the best, while the farthest tube

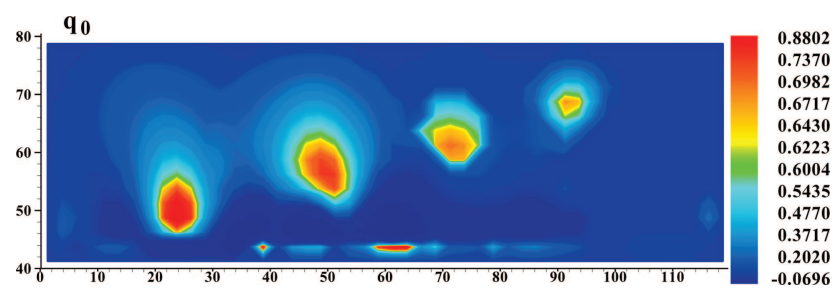

Fig. 4. Slice $y=25 \mathrm{~mm}$ showing the reconstructed quantum yield distribution at $\omega=0$. 
appears to be smaller and slightly closer to the camera than it should be. This implies that spatial resolution significantly improves if other sides of the phantom were imaged. The true value of the quantum yield in the tubes was 0.95 . Therefore, the quantum yield was found with approximately 10\%-20\% error depending on the target's location.

Lifetime can be reconstructed independently from the quantum yield. Thus, having found the complex function $Q$ for any given frequency we exclude the quantum yield from equations

$$
\begin{aligned}
& \operatorname{Re} Q=q_{0} \frac{\operatorname{Re} g_{v^{*}}(\omega)+\omega \tau \operatorname{Im} g_{v^{*}}(\omega)}{1+(\omega \tau)^{2}}, \\
& \operatorname{Im} Q=q_{0} \frac{\operatorname{Im} g_{v^{*}}(\omega)-\omega \tau \operatorname{Re} g_{v^{*}}(\omega)}{1+(\omega \tau)^{2}},
\end{aligned}
$$

and obtain

$$
\tau \simeq \frac{A B \omega}{B^{2} \omega^{2}+\varepsilon}
$$

where

$$
\begin{aligned}
& A=\operatorname{Im} g_{v^{*}}(\omega) \operatorname{Re} Q-\operatorname{Re} g_{v^{*}}(\omega) \operatorname{Im} Q, \\
& B=\operatorname{Re} g_{v^{*}}(\omega) \operatorname{Re} Q+\operatorname{Im} g_{v^{*}}(\omega) \operatorname{Im} Q,
\end{aligned}
$$

and $\varepsilon$ is a regularization parameter. Note that, having found $\tau$, this procedure allows one to recover the quantum yield by use of Eq. (29) or Eq. (30).

Reconstructed images of the function $Q$ at $\omega=$ $1 \mathrm{GHz}$ are shown in Fig. 5. Note that both $\operatorname{Re} Q$ and $\operatorname{Im} Q$ are negative, which was caused by the time delay $t_{d} \simeq 1 \mathrm{~ns}$ in the gating function. The lifetime shown in Fig. 6 was recovered from Eq. (31). The true value of the lifetime in the tubes was $4.05 \times 10^{-9} \mathrm{~s}$. As a result, the reconstruction of the lifetime distri-

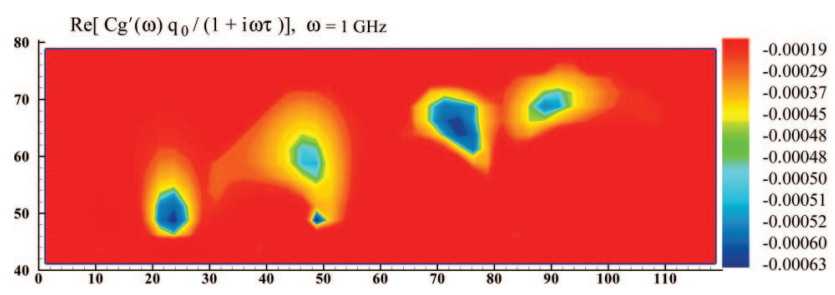

$\operatorname{Im}\left[\mathrm{Cg}^{\prime}(\omega) \mathrm{q}_{0} /(1+\mathrm{i} \omega \tau)\right], \omega=1 \mathrm{GH}$

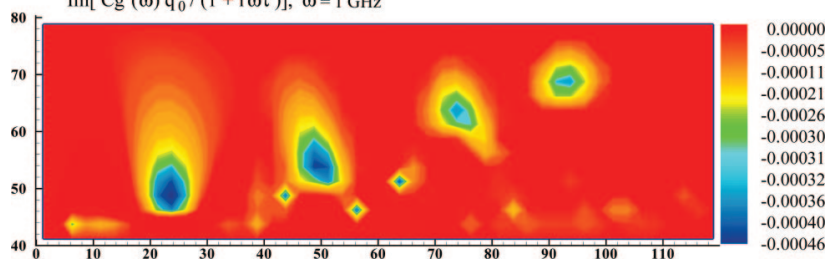

Fig. 5. Reconstructed real and imaginary parts of the function $Q(\mathbf{r} ; \omega)$ at $\omega=1 \mathrm{GHz}$.

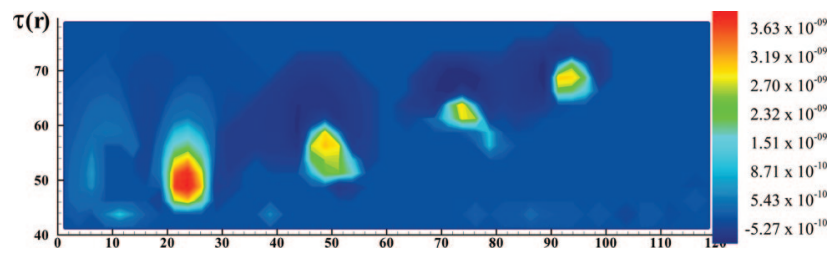

Fig. 6. Reconstructed lifetime distribution from real and imaginary parts of the function $Q(\mathbf{r} ; \omega)$ at $\omega=1 \mathrm{GHz}$.

bution was achieved with relatively good accuracy with approximately 10\%-20\% error.

In summary we have demonstrated the feasibility of fluorescence lifetime imaging experimentally on the basis of a time-gating technique. The time-gating technique provides information about fluorophor distributed inside the studied object in the nanosecond time scale, which facilitates the reconstruction algorithm and allows the fluorescence lifetime distribution to be recovered with relative ease and acceptable accuracy. It provides enough data points in the time domain needed for application of the Fourier transform. The use of the Fourier transform is essential for the time deconvolution of the system of the integral equations employed in the reconstruction. The application of the telegraph equation allows a sufficiently broad interval of frequencies to be covered. Reconstructed image, which is a complex function, contains enough information needed for recovering the lifetime distribution in a sample for any given frequency within the megahertzgigahertz band. This technique was applied in reconstructing the quantum yield and lifetimes of four tubes filled with Rhodamine 6G embedded inside a highly scattering slab. Relatively accurate reconstruction demonstrates the effectiveness and the potential of the proposed technique.

This work was supported in part by a DTI Beacon project and an EU Framework VI project: Integrated Technologies for in vivo Molecular Imaging LSHGCT-2003-503259.

\section{References}

1. E. Shives, Y. Xu, and H. Jiang, "Fluorescence lifetime tomography in turbid media based on an oxygen-sensitive dye," Opt. Express 10, 1557-1562 (2002).

2. V. Y. Soloviev, D. F. Wilson, and S. A. Vinogradov, "Phosphorescence lifetime imaging in turbid media: the inverse problem and experimental image reconstruction," Appl. Opt. 43, 564574 (2004).

3. S. V. Apreleva, D. F. Wilson, and S. A. Vinogradov, "Tomographic imaging of oxygen by phosphorescence lifetime," Appl. Opt. 45, 8547-8559 (2006).

4. S. Lam, F. Lesage, and X. Intes, "Time domain fluorescence diffuse optical tomography: analytical expressions," Opt. Express 13, 2263-2275 (2005).

5. A. T. N. Kumar, J. Skoch, B. J. Bacskai, D. A. Boas, and A. K. Dunn, "Fluorescence-lifetime-based tomography for turbid media," Opt. Lett. 30, 3357-3349 (2005).

6. A. T. N. Kumar, S. B. Raymond, G. Boverman, D. A. Boas, and B. J. Bacskai, "Time resolved fluorescence tomography of turbid media based on lifetime contrast," Opt. Express 25, 1225512270 (2006). 
7. M. S. Patterson and B. W. Pogue, "Mathematical model for time-resolved and frequency-domain fluorescence spectroscopy in biological tissues," Appl. Opt. 33, 1963-1974 (1994).

8. M. A. O'Leary, D. A. Boas, X. D. Li, B. Chance, and A. G. Yodh, "Fluorescence lifetime imaging in turbid media," Opt. Lett. 21, 158-160 (1996).

9. E. M. Sevick-Muraca, G. Lopez, J. S. Reynolds, T. L. Troy, and C. L. Hutchinson, "Fluorescence and absorption contrast mechanism for biomedical optical imaging using frequency domain techniques," Photochem. Photobiol. 66, 55-64 (1997).

10. J. S. Reynolds, C. A. Thompson, K. J. Webb, F. P. LaPlant, and D. Ben-Amotz, "Frequency domain modeling of reradiation in highly scattering media," Appl. Opt. 36, 2252-2259 (1997).

11. A. B. Milstein, S. Oh, K. J. Webb, C. A. Bouman, Q. Zhang, D. A. Boas, and R. P. Millane, "Fluorescence optical diffusion tomography," Appl. Opt. 42, 3081-3094 (2003).

12. A. Joshi, W. Bangerth, K. Hwan, J. C. Rasmussen, and E. M. Sevick-Muraca, "Fully adaptive FEM based fluorescence tomography from time-dependant measurements with area illumination and detection," Med. Phys. 33, 1299-1310 (2006).

13. J. Siegel, D. S. Elson, S. E. D. Webb, K. C. B. Lee, A. Vladas, G. L. Gambaruto, S. Leveque-Fort, M. J. Lever, P. J. Tadrous, G. W. H. Stamp, A. L. Wallace, A. Sandison, T. F. Watson, F. Alvarez, and P. M. W. French, "Studying biological tissue with fluorescence lifetime imaging: microscopy, endoscopy, and complex decay profiles," Appl. Opt. 42, 2995-3004 (2003).

14. D. Elson, J. Requejo-Isidro, I. Munro, F. Reavell, J. Seigel, K. Suhling, P. Tadrous, R. Benninger, P. Lanigan, J. McGinty, C. Talbot, B. Treanor, S. Webb, A. Sandison, A. Wallace, D. Davis, J. Lever, M. Neil, D. Phillips, G. Stamp, and P. French, "Timedomain fluorescence lifetime imaging applied to biological tissue," Photochem. Photobiol. Sci. 3, 795-801 (2004).

15. V. Y. Soloviev, "Mesh adaptation technique for Fourier-domain fluorescence lifetime imaging," Med. Phys. 33, 4176-4183 (2006).

16. F. Gao, H. Zhao, Y. Tanikawa, and Y. Yamada, "A linear, featured-data scheme for image reconstruction in time-domain fluorescence molecular tomography," Opt. Express 14, 71097124 (2006).
17. V. Soloviev, D. Wilson, and S. Vinogradov, "Phosphorescence lifetime imaging in turbid media: the forward problem," Appl. Opt. 42, 113-123 (2003).

18. D. J. Durian and J. Rundick, "Photon migration at short times and distances and in case of strong absorption," J. Opt. Soc. Am. 14, 235-245 (1997).

19. J. Ripoll, R. B. Schultz, and V. Ntziachristos, "Free-space propagation of diffuse light: theory and experiments," Phys. Rev. Lett. 91, 103901 (2003).

20. R. B. Schulz, J. Peter, W. Semmler, C. D'Andrea, G. Valentini, and R. Cubeddu, "Comparison of noncontact and fiber-based fluorescence-mediated tomography," Opt. Lett. 31, 769-771 (2006).

21. V. V. Sobolev, A Treatise on Radiative Transfer (Van Nostrand, 1963).

22. S. Arridge, "Optical tomography in medical imaging," Inverse Probl. Eng. 15, R41-R93 (1999).

23. V. Y. Soloviev and L. V. Krasnosselskaia, "Consideration of a spread-out source in problems of near-infrared optical tomography," Appl. Opt. 45, 4765-4775 (2006).

24. V. Y. Soloviev and L. V. Krasnosselskaia, "Dynamically adaptive mesh refinement technique for image reconstruction in optical tomography," Appl. Opt. 45, 2828-2837 (2006).

25. H. W. Engl, M. Hanke, and A. Neubauer, Regularization of Inverse Problems (Kluwer Academic, 2000).

26. M. Firbank, "The design, calibration, and usage of a solid scattering and absorbing phantom for near-infrared spectroscopy," Ph.D. dissertation (University College, London, 1994).

27. K. Tahir and C. Dainty, "Experimental measurements of light scattering from samples with specified optical properties," J. Opt. A, Pure Appl. Opt. 7, 207-214 (2005).

28. F. Bohren, "Recurrence relations for the Mie scattering coefficients," J. Opt. Soc. Am. A 4, 612-613 (1987).

29. C. Dunsby, P. M. P. Lanigan, J. McGinty, D. S. Elson, J. Requejo-Isidro, I. Munro, N. Galletly, F. McCann, B. Treanor, B. Onfelt, D. M. Davis, M. A. A. Neil, and P. M. W. French, "An electronically tunable ultrafast laser source applied to fluorescence imaging and fluorescence lifetime imaging microscopy," J. Phys. D 37, 3296-3303 (2004). 